\title{
Can Very High Frequency Instantaneous Pulse Rate Variability Serve as an Obvious Indicator of Peripheral Circulation?
}

\author{
Po-Hsun Huang ${ }^{1}$, Chia-Chi Chang ${ }^{2,3}$, Chin-Yi Huang ${ }^{4}$ and Tzu-Chien Hsiao ${ }^{2,5}$ \\ 1. Institute of Computer Science and Engineering, National Chiao Tung University, Hsinchu, Taiwan, R.O.C. \\ 2. Institute of Biomedical Engineering, National Chiao Tung University, Hsinchu, Taiwan, R.O.C. \\ 3. A-Neuron Electronic Corporation, Hsinchu, Taiwan, R.O.C. \\ 4. Yo-Yo Pediatric Clinic, Kaohsiung, Taiwan, R.O.C. \\ 5. Department of Computer Science, National Chiao Tung University, Hsinchu, Taiwan, R.O.C.
}

\begin{abstract}
HRV (heart rate variability) is a general noninvasive method for indicating the activities of ANS (autonomic nervous system). The surrogate of HRV is PRV (pulse rate variability), where the variability of resting PPG (photo plethysmo graphy) indicates the activities of ANS and peripheral circulation. However, the quick responses of thermoregulation and limb movement are restrictedly performed since of the beat-to-beat timescale property of PRV. Recently, iPRV (instantaneous pulse rate variability) has been developed to break the limitation. The iPRV adopts empirical mode decomposition for noise reduction and estimates the instantaneous period for higher time resolution. Thus, an ultra-frequency band called VHF (very high frequency) was held. The aim of this study is to concentrate the iPRV presentation on fever adolescent who is possibly under immature body regulation. Thirty participates, whose age were 7 to 18 years old, were recruited in pediatric clinic. The resting PPG signals were acquired for 10 minutes in a supine position. The analysis results show the conventional ranges of iPRV also observe the activities of ANS. As well, the modified indices including VHF are significant difference ( $p$-value $<0.05$ ) between fever and feverless symptoms. Based on the statistical evidence, this study provides the potential of the indicator of thermoregulation on peripheral circulation.
\end{abstract}

Key words: Peripheral circulation, instantaneous pulse rate variability, very high frequency band.

\section{Introduction}

HRV (heart rate variability) is generally a common and widely non-invasion used method for indicating the activities of ANS (autonomic nervous system) [1]. ECG (Electrocardiography) signal is used to observe the activities of heart. The interval between each R-peak on ECG signal called RRI is used to calculate the variability of heart beats. For frequency domain analysis, it is usually transformed into power spectrum by FFT (fast Fourier transform) after interpolating the RRI [2] (Fig.1 (a)). The SNS (sympathetic activities) and PNS (parasympathetic activities) are two main

Corresponding author: Tzu-Chien Hsiao, Ph.D., associate professor, research fields: biomedical signals analysis, virtual biomedical instrumentation. parts of ANS. Previous study showed the short-term HRV spectrum contained two major frequency bands, which are used as the indicator of ANS. Low-frequency (LF, 0.04 0.15 Hz) presents the activities of SNS and high-frequency (HF, 0.15 0.4 $\mathrm{Hz}$ ) is mainly regulated by PNS [2]. However, the use of ECG is not convenient at home or clinic. Besides it, the ECG signal only has central circulation information. An alternative measurement of HRV, called PRV (pulse rate variability) was proposed. PRV replaces ECG recording in HRV with PPG (photoplethysmography) (Fig. 1(b)). There is the PTT (pulse transit time) between the R-peak and pulse wave, which is resulting from complex regulation of cardiac pumping function, respiratory movement, and 


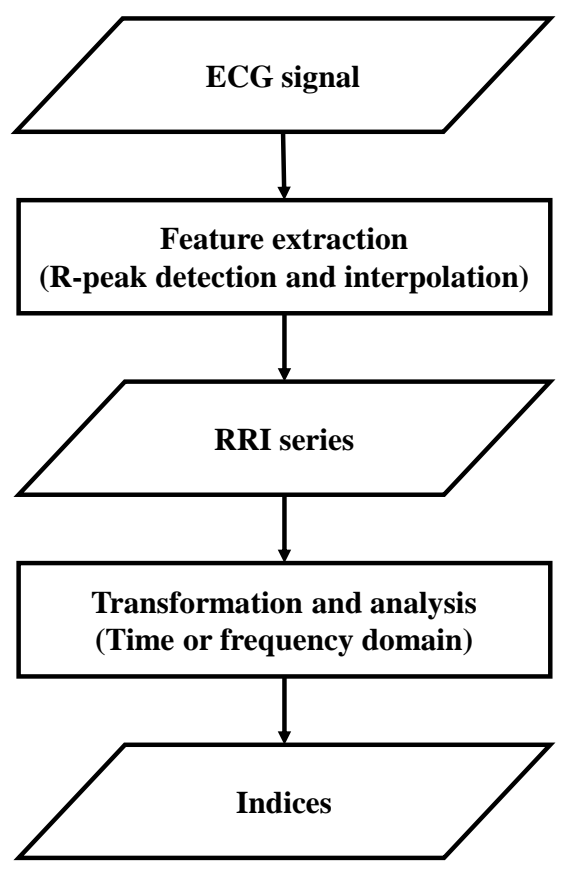

(a)

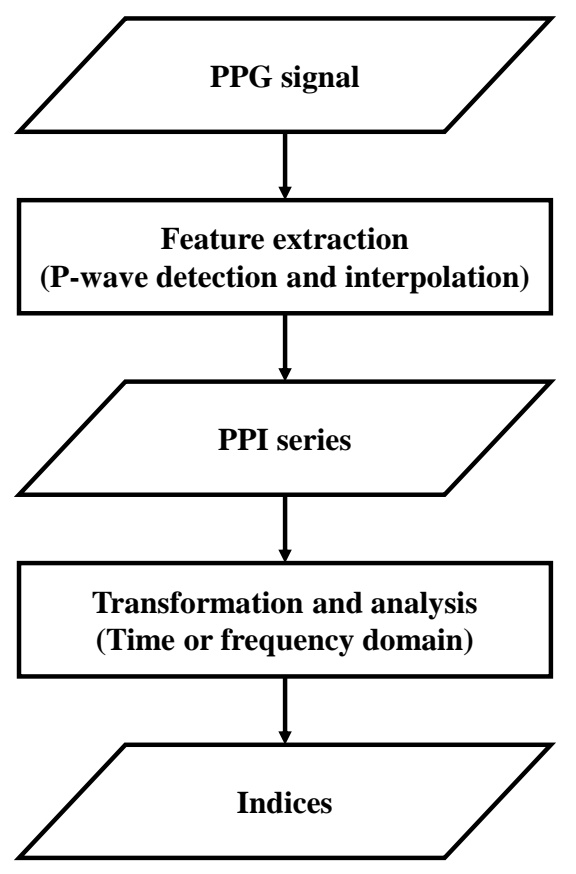

(b)

Fig. 1 The procedure of (a) HRV and (b) PRV.

vasculartone, PRV is still capable to assess the ANS activities during some non-stationary conditions [3]. Though the PPG signal has potential to provide more information about peripheral circulation [4], it is less to be indicated by PRV.

However, conventional frequency-domain analysis of HRV and PRV has several limitations. The time resolution of HRV is limited by tachogram, which adopted the interpolation method in spectral analysis [5]. For breaking the limitation, iPRV (instantaneous pulse rate variability) was proposed [6]. iPRV adopted PRV technique and applied the frequency range extension method based on HHT (hilbert-huang transform) [7]. HHT can deal with the non-stationary and nonlinear data by its pre-process, called EMD (empirical mode decomposition). However, the intermittency phenomenon, which is called mode mixing problem is involved in EMD. The noise-assisted method called EEMD (ensemble EMD) was proposed for solving this problem [8]. In iPRV spectral analysis, the extended frequency band called very high frequency (VHF, 0.4 0.9 $\mathrm{Hz}$ in this study) was proposed. Several studies used head-up tilt or passive lag raising test to stimulate SNS or change blood volume for observing the change in power of VHF $[6,9,10]$.

Body temperature, which usually refers to the rectal temperature in animals, is an important homeothermic autoregulation of humans. In many mammals, the POA (preoptic area), a region of hypothalamus, is considered as the thermoregulatory center. The normal body temperature is about $36.5 \sim 37.5{ }^{\circ} \mathrm{C}$ and it is effected by the environment temperature. When the receptor receives an environment temperature change, POA controls some organs activities or reaction to regulate the body temperature, and then the body temperature also sends feedback to POA for maintaining the body temperature. Sometimes, an abnormal rise in body temperature occurs, which is higher than normal body temperature, and we call it fever. It is usually caused by infection of bacterium or virus. When a person gets fever, the hypothalamus changes the set point to the higher temperature and it makes the body feel cold because the body temperature is lower than the set point. Therefore, POA tries to rise the body temperature [11, 12]. 
There are many mechanisms to regulate the body temperature, for instance cutaneous vasomotion, blood pressure increases. The peripheral circulation always makes quick response to the thermoregulation [13, 14]. Nevertheless, it is restrictedly performed since of the beat-to-beat timescale of HRV or PRV. So can the VHF of iPRV be an indicator of the thermoregulation on peripheral circulation?

The body regulation has much difference between fever patients and normal body temperature people. Therefore, this study recruited different body temperature subjects to indicate the different body regulation of them by iPRV spectral analysis. The paper is organized as follows: Section 2 introduces the recruited subjects and iPRV procedure. Section 3 presents the results calculated by iPRV spectral analysis. Section 4 compares the iPRV results with the conventional method (PRV). Section 5 discusses the thermoregulation and the results. Section 6 gives conclusions. Section 7 presents the further work.

\section{Material and Method}

\subsection{Subjects and Experiment}

The experiment was carried out in Yo-Yo Clinic, Kaohsiung, Taiwan. The body temperature was measured by ear thermometer (Radiant TH889, Radiant Innovation Inc.) and the signal was acquired by PPG (Nonin 8500, Nonin Medical Inc.) with 200 $\mathrm{Hz}$ sampling rate.

30 subjects aged 7 to 18 years old were recruited in this study. 15 subjects whose body temperature is higher than $37.9^{\circ} \mathrm{C}$ serve as Fever group, others serve as Control group. The body temperature of all subjects were measured before acquiring the PPG signal. The subject was required to rest quietly in a supine position when the PPG signal acquire 10 minutes. This experiment was approved by the Research Ethics Committee for Human Subject Protection of the National Chiao Tung University. Informed consent was obtained from all subjects before the experiment.

\section{2 iPRV Procedure}

Firstly, PPG data is decomposed into ordered IMFs (intrinsic mode functions) by using CEEMD (complementary EEMD) [15]. The definition of IMF is: (1) the number of local extrema must be equal to the number of zero-crossings or differ at most by one and (2) at any point, the value of average envelop must approximately equal to zero. The CEEMD adopts the EMD technique and improves the EEMD for not only solving the mode mixing problem but reducing the influence of white noise, i.e. finding the local extrema from the input PPG signal $x(t)$ and using cubic spline to interpolate the local maximum and local minimum to generate the upper envelope $\mathrm{U}(\mathrm{t})$ and lower envelope $\mathrm{L}(\mathrm{t})$, respectively. The average envelope $M(t)$ is calculated by the mean of $U(t)$ and $\mathrm{L}(\mathrm{t})$.

$$
M(t)=\frac{U(t)+L(t)}{2}
$$

And the output of this process is calculated as follows.

$$
H(t)=x(t)-M(t)
$$

The above steps are called sifting process. After this process, it needs to determine the output $\mathrm{H}(\mathrm{t})$ is an IMF or not. If $\mathrm{H}(\mathrm{t})$ is not an IMF, use $\mathrm{H}(\mathrm{t})$ to replace the $\mathrm{x}(\mathrm{t})$ to redo the sifting process. If $\mathrm{H}(\mathrm{t})$ is an IMF, output the $\mathrm{H}(\mathrm{t})$ as the $\operatorname{IMF}(\mathrm{t})$ and redo the EMD by using the difference between $x(t)$ and $\operatorname{IMF}(t)$ as the new input. The EMD is finished until satisfying the criteria. The EEMD is similar to EMD but adds white noise before doing sifting process. It needs to do many times EMD and average all correspond output IMFs to ensemble IMFs for reducing the influence of white noise. However, the effect of white noise remains to influent the output. Therefore, the CEEMD was used in this study. In the CEEMD, the white noise $\mathrm{N}(\mathrm{t})$ is added in paired to the input data $\mathrm{x}(\mathrm{t})$ to generate two sets of ensemble IMFs. Therefore, the new inputs $x p(t)$ and $x n(t)$ composed the white noise and original input are calculated as: 


$$
\left[\begin{array}{c}
x_{p}(t) \\
x_{n}(t)
\end{array}\right]=\left[\begin{array}{cc}
1 & 1 \\
1 & -1
\end{array}\right]\left[\begin{array}{c}
x(t) \\
N(t)
\end{array}\right]
$$

After the decomposition, according to the previous study [6], the one of IMFs which is sinusoid-like and the peaks correspond to the pulse peaks of input PPG signal is determined as a main component IMFmain. In order to break the timescale limitation, the normalized IMFmain (empirical frequency modulation signal, $\mathrm{F}(\mathrm{t})$ ) is calculated into the instantaneous frequency (iFreq) and iPeriod (instantaneous period) by using NDQ (normalized direct quadrature) [16] and inverse, respectively. The $\mathrm{F}(\mathrm{t})$ is now assumed to be a cosine function and its quadrature, sine function, can be calculated directly. Therefore, instantaneous phase, $\phi(t)$, can be computed as Eqs. (4) and (5).

$$
\begin{gathered}
\sin \phi(t)=\sqrt{1-F^{2}(t)} \\
\phi(t)=\arctan \left(\frac{\sqrt{1-F^{2}(t)}}{F(t)}\right)
\end{gathered}
$$

Then the iFreq is obtained from the derivative of $\phi(t)$, as

$$
i \operatorname{Freq}(t)=\frac{1}{\sqrt{1-F^{2}(t)}} \frac{\partial F(t)}{\partial t}
$$

Finally, the iPeriod is estimated by inversion of iFreq in order to indicate the time series of heartbeat rhythm as RRI. After calculating iPeriod, FFT was used to transform the iPeriod data into frequency domain for further power spectrum analysis. The processing flowchart and corresponding demonstrations of iPRV procedure are shown in Fig. 2.

\subsection{Index Calculating}

The standard of bandwidth of LF is between 0.04 $\mathrm{Hz}$ and $0.15 \mathrm{~Hz}$ and $\mathrm{HF}$ is between $0.15 \mathrm{~Hz}$ and 0.4 $\mathrm{Hz}$ [2] (Fig. 3). The conventional indices of normalized power (nLF and nHF) and LF-HFratio are calculated as follows:

$$
\begin{aligned}
& \mathrm{nLF}^{b}=100 \% * \frac{\mathrm{LF}}{\mathrm{LF}+\mathrm{HF}} \\
& \mathrm{nHF}^{b}=100 \% * \frac{\mathrm{HF}}{\mathrm{LF}+\mathrm{HF}} \\
& \mathrm{LF}-\mathrm{HF}_{\text {ratio }}=100 \% * \frac{\mathrm{LF}}{\mathrm{HF}}
\end{aligned}
$$

For calculating including VHF, we assume the bandwidth of VHF of iPRV is $0.4 \sim 0.9 \mathrm{~Hz}$ instead of 0.4 0.5 Hz in PRV (Fig. 3). The modified indices are calculated as follows:
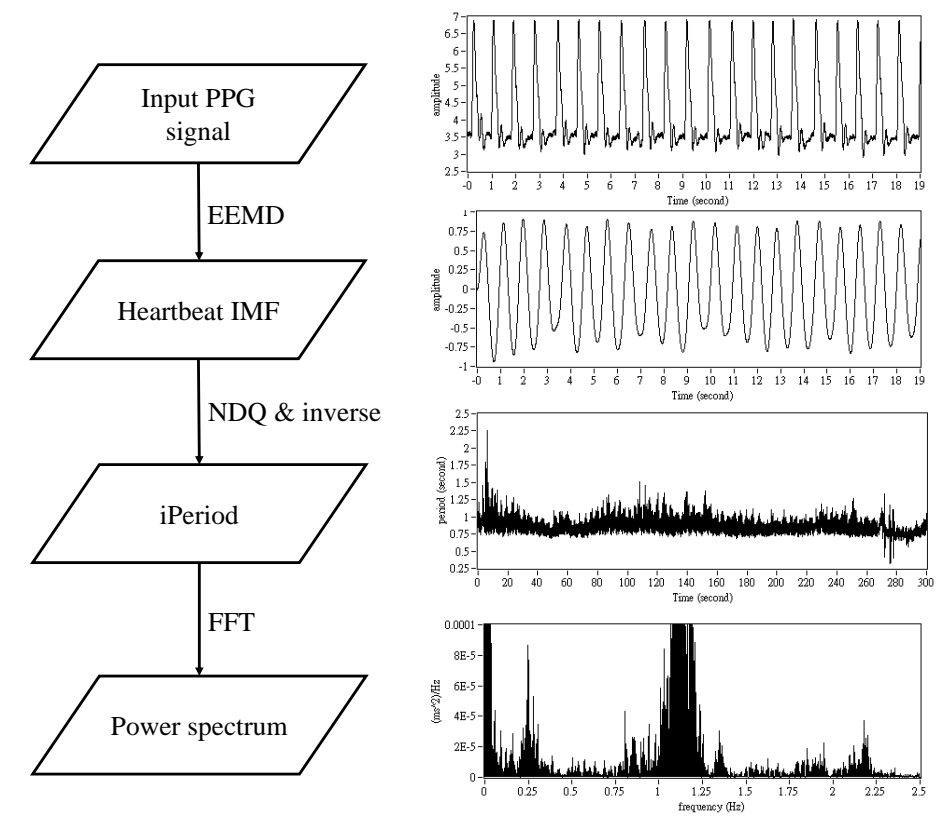

Fig. 2 The processing flowchart and corresponding demonstration of iPRV procedure. 


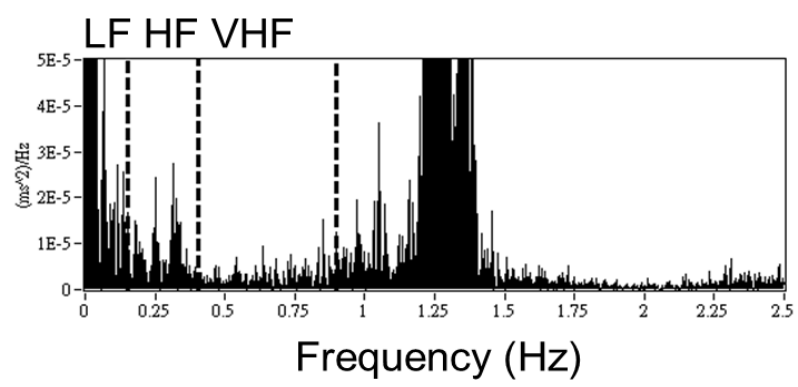

Fig. 3 The frequency band of power spectrum.

$$
\begin{aligned}
\mathrm{nLF}^{a} & =100 \% * \frac{\mathrm{LF}}{\mathrm{LF}+\mathrm{HF}+\mathrm{VHF}} \\
\mathrm{nHF}^{a} & =100 \% * \frac{\mathrm{HF}}{\mathrm{LF}+\mathrm{HF}+\mathrm{VHF}} \\
\mathrm{nVHF}^{a} & =100 \% * \frac{\mathrm{VHF}}{\mathrm{LF}+\mathrm{HF}+\mathrm{VHF}}
\end{aligned}
$$

Each abbreviation, LF, HF and VHF in equations present the power summation of corresponding bands.

\section{Result}

The results of spectral analysis between the two groups are shown in Table 1. In frequency domain, the $\mathrm{nLFb}$ values in Fever group and Control group are $0.63 \pm 0.13$ and $0.47 \pm 0.16$, respectively. And these two groups are significantly different $(p<0.05)$. The LF-HFratio in Fever group and Control group are $2.08 \pm 1.36$ and $1.1 \pm 0.87$, respectively. These group are also significant difference $(p<0.05)$. On the other hand, $\mathrm{nHFb}$ values in Fever group $(0.38 \pm 0.13)$ are significantly lower than those in Control group $(0.54 \pm 0.16, p<0.05)$. If calculating the normalized power including VHF, nHFa values in Fever group
$(0.19 \pm 0.07)$ are significantly lower than those in Control group $(0.31 \pm 0.12, \mathrm{p}<0.05)$. In the $\mathrm{nLFa}$ values and $\mathrm{nVHFa}$ values a trend can be observed, the mean value of $\mathrm{nLFa}$ in Fever group $(0.33 \pm 0.12)$ is higher than those in Control group $(0.26 \pm 0.11)$ and the mean value of $n V H F a$ in Fever group $(0.49 \pm 0.14)$ is lower than those in Control group (0.43 \pm 0.1$)$.

\section{4. iPRV Result Compared with PRV}

The comparison results between PRV and iPRV are shown in Table 2.

There are no significant difference in $\mathrm{nLFb}$ and nHFb between PRV and iPRV. While including VHF, all indices in PRV are significantly different from the one of iPRV whether in Control group or Fever group. However, indices whether including VHF or not, have the same trend in PRV and iPRV. For example, comparing to Control group, $\mathrm{nLFb}$ increased in Fever group whether calculated by PRV or iPRV. Nevertheless, the value of $n V H F a$ in iPRV is much larger than the value in PRV.

The conventional indices, $\mathrm{nLFb}, \mathrm{nHFb}$ and LF-HFratio, calculated by HRV usually present the activities of ANS [1,2]. The PRV can be used as an alternative measurement of the HRV [3]. So $\mathrm{nLFb}$, $\mathrm{nHFb}$ and LF-HFratio are useful for indicating the activities of ANS by PRV. The results of this study also show the value of $n L F b, n H F b$ and LF-HFratio calculated by iPRV have no significant difference to PRV. Therefore, $\mathrm{nLFb}, \mathrm{nHFb}$ and LF-HFratio calculated

Table 1 The result between two groups.

\begin{tabular}{lll}
\hline$(\%)$ & Control group & Fever group \\
\hline $\mathrm{nLFa}$ & $26.4 \pm 10.9$ & $32.5 \pm 11.7$ \\
$(0.04 \sim 0.15 \mathrm{~Hz})$ & $30.5 \pm 11.5^{*}$ & $18.8 \pm 7.4$ \\
$\mathrm{nHFa}$ & $43.1 \pm 10.4$ & $48.7 \pm 13.6$ \\
$(0.15 \sim 0.40 \mathrm{~Hz})$ & $46.5 \pm 16.4^{*}$ & $62.5 \pm 12.8$ \\
$\mathrm{nVHFa}$ & & $37.5 \pm 12.8$ \\
$(0.40 \sim 0.90 \mathrm{~Hz})$ & $53.5 \pm 16.4^{*}$ & $207.5 \pm 136.2$ \\
$\mathrm{nLFb}$ & $109.9 \pm 86.9^{*}$ & \\
nHFb & $0.15 \mathrm{~Hz})$ &
\end{tabular}

The form is mean \pm standard deviation and $*$ means $p$-value $<0.05$ compared with Fever group. 
Table 2 The comparison between PRV and iPRV.

\begin{tabular}{lllll}
\hline & \multicolumn{2}{c}{ Control group } & Fever group \\
\cline { 2 - 4 }$(\%)$ & PRV & iPRV & PRV & iPRV \\
\hline $\mathrm{nLFa}$ & $42.8 \pm 15.8^{* \#}$ & $26.4 \pm 10.9$ & $56.7 \pm 11.9 \#$ & $32.5 \pm 11.7$ \\
$\mathrm{nHFa}$ & $43.9 \pm 16.6^{*} \#$ & $30.5 \pm 11.5^{*}$ & $27.5 \pm 10.1 \#$ & $18.8 \pm 7.4$ \\
$\mathrm{nVHFa}$ & $13.2 \pm 10.2 \#$ & $43.1 \pm 10.4$ & $15.8 \pm 6.8 \#$ & $48.7 \pm 13.6$ \\
$\mathrm{nLFb}$ & $49.6 \pm 16.6^{*}$ & $46.5 \pm 16.4^{*}$ & $67.2 \pm 12.4$ & $62.5 \pm 12.8$ \\
$\mathrm{nHFb}$ & $50.4 \pm 16.6^{*}$ & $53.5 \pm 16.4^{*}$ & $32.8 \pm 12.4$ & $37.5 \pm 12.8$ \\
LF-HFratio & $128.8 \pm 112.6^{*}$ & $109.9 \pm 86.9^{*}$ & $263.6 \pm 187.0$ & $207.5 \pm 136.2$ \\
\hline
\end{tabular}

The form is mean \pm standard deviation, * means $p$-value $<0.05$ compared with Fever group, \# means $p$-value $<0.05$ compared with PRV.

by iPRV can also indicate the activities of ANS. For modified indices in this study, because of the narrow bandwidth of VHF in PRV, nLFa and nHFa are similar to the conventional indices. Otherwise, the power of VHF is much larger in iPRV. VHF band may contain more information about not only activity of ANS but more body regulation.

\section{5. iPRV Result and Thermoregulation}

Heart activities are usually dominated by ANS. It is considered that the heart rate increases because the SNS is more excitatory than the PNS. On the contrary, the heart rate decreases because the PNS is more excitatory than the SNS. The conventional HRV analysis in frequency domain is usually used for the ANS activity observation. The $\mathrm{nLFb}$ and $\mathrm{nHFb}$ are usually considered as the activities of SNS and PNS. The LF-HFratio is considered as the regulation of SNS [1, 2]. The correlation of iPRV and HRV in the frequency domain analysis during non-stationary conditions has been examined in LF and HF [6, 17-19]. HRV analysis in frequency domain rarely uses VHF because of the resolution of time series, but iPRV breaks the restriction and provides much wider frequency band for mining.

The ANS rises the body temperature when someone has fever [11, 12]. Therefore, the SNS is more excitatory than the PNS. Regarding the results in Table 1, the value of nLFb and LF-HFratio in Fever group is significantly higher than Control group. This means that the SNS is actually more excitatory in fever patients.

If calculating the normalized power including VHF, the nHFa in Fever group are significantly lower than those in Control group. There is a trend that the mean value of the $\mathrm{nLFa}$ and $\mathrm{nVHFa}$ is larger in Fever group, though this is not significant. Maybe the nLFa and the nHFa do not only represent the activities of SNS and PNS, but have more regulation mixed in them. For VHF, maybe there is too much information to indicate the difference between the two groups. The meaning of these normalized powers needs further research to examine.

For the extended frequency band, VHF proposed by iPRV, previous studies have assumed that VHF has possible meaning of cardiac output or peripheral circulation $[6,9]$. Another study also showed the VHF band has potential to evaluate fluid responsiveness [10]. In this study, the fever patients have the constriction of peripheral blood vessels, and it increases blood pressure. These peripheral regulation cannot be observed in LF and HF by conventional HRV analysis. So, the VHF band might also contain information of regulation between the peripheral blood vessels and blood pressure, and the normalized power calculated including VHF might contain the regulation of ANS and peripheral circulation. The bandwidth of VHF is very wide ( 0.4 to $0.9 \mathrm{~Hz}$ ), it is possible to find more useful information by separating the band into more numbers of narrow bandwidth. It also needs further research and investigation for verification. 


\section{Conclusion}

The aim of this study is to concentrate the iPRV presentation on fever adolescent with immature body regulation. Conventional evaluating indicators of ANS and peripheral circulation remain restrictedly performed since of the beat-to-beat timescale property of PRV. Therefore, iPRV method of this study adopts CEEMD and NDQ method for instantaneous period calculating to break through the limitation. The results show the conventional range of iPRV also observe the activities of ANS. As well, the modified indices including VHF have significant difference between fever and feverless symptoms. There are many different peripheral circulations between fever patients and feverless people. This is the first time to provide the potential indicator of thermoregulation on peripheral circulation. However, it still needs more experiments for further approval.

\section{Further Work}

There are many approaches and affects that cause the body temperature increasing, but it did not include in this study. Further comparison is needed for the corresponding responses of the body temperature increasing. For example, a person after exercise or fever patients get higher body temperature than the person during resting or the patient in normal situation. The regulation mechanisms of body are different. Comparing between the people who have fever and after exercise probably may find the physiological meaning of VHF, especially the regulation of peripheral circulation. Moreover, the PTT indicates the delay between R-peak and pulse wave, the delay time is also influenced by vascular tone or cardiac pumping function, which had been shown in the previous studies that VHF has potential to indicate them. Therefore, to compare the difference between iPRV and HRV under different body temperature is also an important point of the following research.

\section{Acknowledgement}

This work was fully supported by Taiwan Ministry of Science and Technology under grant number MOST-105-2221-E-009-159 and MOST 105-2634-E009-003, and in part by "Aim for the Top University Plan" of National Chiao Tung University and Ministry of Education, Taiwan, R.O.C.

\section{References}

[1] Akselrod, S., Gordon, D., Ubel, F. A., Shannon, D. C., Berger, A. C., and Cohen, R. J. 1981. "Power Spectrum Analysis of Heart Rate Fluctuation: A Quantitative Probe of Beat-to-Beat Cardiovascular Control." Science 213: 220-22.

[2] Malik, M. 1996. "Heart Rate Variability: Standards of Measurement, Physiological Interpretation, and Clinical Use.” European Heart Journal 17: 354-81.

[3] Gil, E., Orini, M., Bailon, R., Vergara, J. M., Mainardi, L., and Laguna, P. 2010. "Photoplethysmography Pulse Rate Variability as a Surrogate Measurement of Heart Rate Variability during Non-stationary Conditions." Physiological Measurement 3: 1271-90.

[4] Allen, J. 2007. "Photoplethysmography and Its Application in Clinical Physiological Measurement." Physiological Measurement 28 (3): R1.

[5] Berntson, G. G., Bigger, Jr. Jt., Eckberg, D. L., Grossman, P., Kaufmann, P. G., Stone, P. H., and Van Der Molen, M. W. 1997. "Heart Rate Variability: Origins, Methods, and Interpretive Caveats." Psychophysiology 34: 623-48.

[6] Chang, C. C., Hsiao, T. C., and Hsu, H. Y. 2014. "Frequency Range Extension of Spectral Analysis of Pulse Rate Variability Based on Hilbert-Huang Transform." Medical \& Biological Engineering \& Computing 52: 343-51.

[7] Huang, N. E., Shen, Z., and Long, S. R., etc. 1998. "The Empirical Mode Decomposition and the Hilbert Spectrum for Nonlinear and Non-Stationary Time Series Analysis." In Proceedings of the Royal Society of London. Series A: Mathematical, Physical and Engineering Sciences, 454: 903-95.

[8] Wu, Z., and Huang, N. E. 2009. "Ensemble Empirical Mode Decomposition: A Noise-Assisted Data Analysis Method." Advances in Adaptive Data Analysis 1: 1-41.

[9] Chang, C. C., Hsu, H. Y., and Hsiao, T. C. 2014. "The Interpretation of Very High Frequency Band of Instantaneous Pulse Rate Variability during Paced Respiration." Biomedical Engineering Online 13: 46-56. 
[10] Lin, P.-C., Chang, C.-C., Hsu, H.-Y., and Hsiao, T.-C. 2016. "Frequency Domain Analysis for Assessing Fluid Responsiveness by Using Instantaneous Pulse Rate Variability." International Journal of Advanced Computer Science and Applications 7 (2): 229-33.

[11] Nakamura, K. 2011. "Central Circuitries for Body Temperature Regulation and Fever." American Journal of Physiology-Regulatory, Integrative and Comparative Physiology 301: 1207-28.

[12] McCorry, L. K. 2007. "Physiology of the Autonomic Nervous System." American Journal of Pharmaceutical Education 71 (78): 1-11.

[13] Burton, A. C. 1939. "The Range and Variability of the Blood Flow in the Human Fingers and the Vasomotor Regulation of Body Temperature." American Journal of Physiology 127: 437-53.

[14] Burton, A. C., and Taylo, R. M. 1940. "A Study of the Adjustment of Peripheral Vascular Tone to the Requirements of the Regulation of Body Temperature." American Journal of Physiology 129: 565-77.

[15] Yeh, J. R., Shieh, J. S., and Huang, N. E. 2010. "Complementary Ensemble Empirical Mode Decomposition: A Novel Noise Enhanced Data Analysis
Method." Advances in Adaptive Data Analysis 2 (2): 135-56.

[16] Huang, N. E., Wu, Z., and Long, S. R. 2009. "On Instantaneous Frequency." Advances in Adaptive Data Analysis 1: 177-229.

[17] Lin, P. C., Hsu, K. C., Chang, C. C., and Hsiao, T. C. 2014. "Reliability of Instantaneous Pulse Rate Variability by Using Photoplethysmography." Presented at the Workshop on Biomedical Microelectronic Translational Systems Research (WBMTSR).

[18] Lin, P. C., Huang, P. H., Chang, C. C., Hsu, H. Y., and Hsiao, T. C. 2015. "A Novel Index of Photoplethysmography by Using Instantaneous Pulse Rate Variability during Non-stationary Condition." Presented at the IEEE International Conference on Consumer Electronics-Taiwan (ICCE-TW), 100-1, doi: 10.1109/ICCE-TW.2015.7216799.

[19] Lin, P. C., Chang, C. C., Hsiao, T. C., and Hsu, H. Y. 2015. "The Circulation Assessment of Daily E-Health by Using Instantaneous Pulse Rate Variability during Nonstationary Conditions." Presented at the 7th International Conference on eHealth, Telemedicine and Social Medicine, (eTELEMED), 209-12, ISBN: 978-1-61208-384-1. 